\title{
All MiNDS on the brain
}

Research on the human brain has uncovered several specific regions that are promising targets for drug-mediated modulation to treat neurologic disorders. But therapeutic options have been limited by a lack of devices that can deliver drugs with high spatial resolution, are refillable and can operate for months or years after implantation in the brain. A new device, from the groups of Robert Langer and Michael Cima at the Massachusetts Institute of Technology, aims to provide a solution to long-standing drug delivery problems ${ }^{1}$.

Systemic drug delivery in diseases of the central nervous system is often associated with serious side effects. This has prompted many research groups to develop alternative systems that release drugs directly in the brain. Catheter-based, refillable devices have enabled sustained delivery of drugs to the center of brain tumors, but not in a controllable manner. Devices that enable controlled release but are not refillable, and devices that can penetrate the surface of the brain with microneedles have also been described. Yet other, more advanced approaches have combined drug delivery with light emission and with monitoring of electrical activity. But these devices were focused on research applications, and, although promising, have not been shown to provide leakage-free delivery of drugs to small regions of the brain and access to deep regions of large animal brains.

The miniaturized neural drug delivery system (MiNDS) from Langer, Cima and colleagues ${ }^{1}$ gets closer to the goal, and combines delivery with recording of electrical activity. The scientists microfabricate a device that incorporates a tungsten electrode, two borosilicate channels and poly(pyromellitic dianhydride-co-4,40-oxydianiline) amic acid templates, aligning them in a stainlesssteel needle. For drug delivery, the MiNDS is connected to pumps whose reservoirs can be refilled, even if implanted, using a needle.

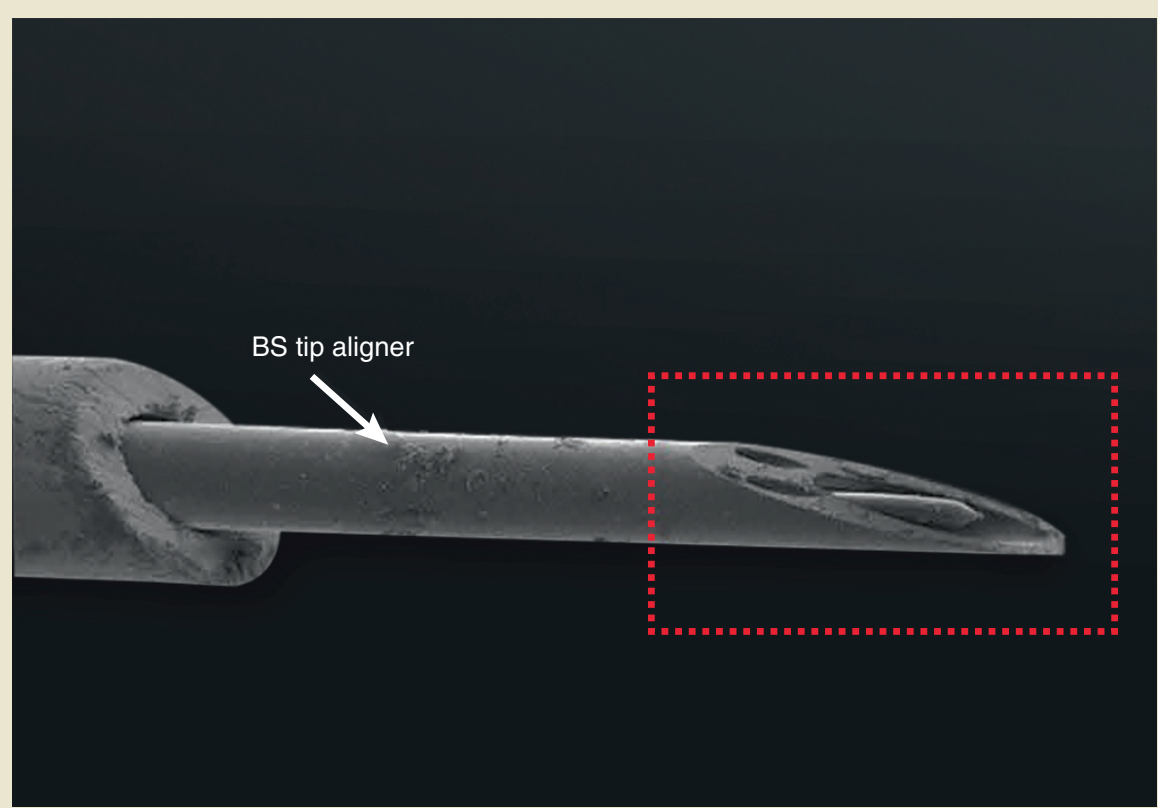

Reproduced with permission from Dagdeviren, C. et al. Sci. Transl. Med. 10, eaan2742 (2018).

"It's an assembly of different technologies into an operating system that can control drug delivery," says Ada Poon, associate professor of electrical engineering at Stanford University. "They are really clever in collecting the different off-the-shelf components from the different vendors and in putting them together with an application in mind," she adds.

Borosilicate and stainless steel are known to be biocompatible with the brain, and the authors demonstrate that eight weeks after implantation in rats, tissue damage and immune activation are limited to the implantation site, suggesting potential longterm implantability. The team constructs long $(10 \mathrm{~cm})$ and short $(1 \mathrm{~cm})$ versions of the MiNDS that have negligible passive passage of fluid and no infusion after pumping is shut off. Bolus delivery is localized to $\sim 1 \mathrm{~mm}$ diffusion in each direction. In rats and non-human primates, both the short and long devices can deliver drugs to deep brain structures and can measure the drugs' effects on neural activity at the single-neuron level.

The utility of the approach has not yet been demonstrated in humans. "It's likely to work," says Poon, "because all the individual components are working by themselves." Another major issue is the battery life of the pumps, which is currently only about a month. The treatment of psychiatric and neurological diseases has not been a rapidly evolving one, but is in dire need of a technology that enables reaching specific brain structures in a controlled manner. Perhaps one is on the way.

Irene Jarchum, Senior Editor

1. Dagdeviren, C. et al. Sci. Transl. Med. 10, eaan2742 (2018). 